\title{
SUGLI ENTI ANALITICI.
}

\author{
Nota di E. Study (Bonn).
}

Adunanza del 25 marzo 1906.

In un lavoro recente il sig. T. Levi-Civita ha indicato una estensione importante di un teorema fondamentale della teoria delle funzioni di una variabile complessa ${ }^{*}$ ).

Qui mi propongo di esporre altre generalizzazioni dello stesso teorema e di teoremi affini, tutti spesso usati, specialmente dal sig. H. A. Schwarz, nei cui scritti si trovano $\mathrm{i}$ casi più semplici $(n=\mathrm{I})$ dei nostri teoremi XVI-XX $\left.{ }^{* *}\right)$.

Queste generalizzazioni sono basate su due idee, l'una, di massima importanza, sviluppata dal sig. C. SEGRE ${ }^{* * *}$ ), l'altra contenuta nel nostro Teorema I. L'idea del SEGRE consiste nella ripetizione del passaggio abituale dal campo reale al campo complesso. Io debbo a lui - mi è grato dichiararlo - non solo questo procedimento, ma anche un numero considerevole di nozioni e di risultati speciali. Il Segre peró si è ristretto alla considerazione degli enti algebrici, ed ha fatto un largo uso di ragionamenti geometrici $\left.{ }^{* * * *}\right)$. Nel caso degli enti analitici, di cui parlerò io, mi pare che manchi ancora una parte delle definizioni necessarie ad esprimere $i$ risultati in forma rigorosa, semplice, ed abbastanza generale. Per esempio, la sola nozione del contenere si deve scindere in almeno tre nozioni differenti, di cui due vengono usate nel presente lavoro. Inoltre riuscirà forse gradito a qualche lettore di veder presentata una tale teoria in maniera puramente analitica. Tutto quello che segue si potrà intendere senza conoscenza della geometria moderna (ed anche, io credo, senz'alcuna difficoltd).

*) T. Levi-Civita, Sulle funzioni di due o piil variabili complesse [Rend. Acc. Lincei, vol. XIV (igo5), $2^{\circ}$ semestre, pp. 492-499].

**) Veggansi le citazioni nell'articolo del sig. Osgood nella Encyklopädie der Mathematischen Wissenschaften, etc., II, B, I, nota $\mathrm{I} 20$ in calce.

***) CoRRado SEGRE, Le rappresentazioni reali delle forme complesse e gli enti iperalgebrici [Math. Annalen, XL (I892), pp. 4r3-467].

****) Pare che per queste ragioni la Memoria del SEGRE non sia conosciuta quanto meriterebbe. Anche nell'articolo citato dell'Osgood (scritto colla più gran diligenza) le idee del SEGRE ven. gono trascurate. Però il SEGRe stesso ha avvertito esplicitamente (alla fine del $n^{\circ} 19$, pag. 442) che parecchie sue considerazioni si possono svolgere, in modo pienamente analogo, per gli enti analitici. 
Cominciamo con qualche definizione ${ }^{*}$ ). Useremo le parole varietà $e$ spazio in sensi differenti.

Considerando i sistemi di $m$ variabili (finite) complesse $\xi_{1}, \ldots \xi_{m}$, parleremo (come si suole) di "punti» di una varietà $V_{m}$, e delle varietà $V_{o}, \ldots V_{m-1}$ analitiche immerse nella $V_{n}$, denotando coll'indice in ciascun caso la "dimensione complessa » dell'ente considerato, cioè il numero delle variabili complesse rimaste indipendenti. Una $V_{\mathrm{o}}$ sarà sempre un punto della $V_{m}$.

Restringendo invece le variabili $\xi_{\mathrm{I}}, \ldots \xi_{m}$ a valori reali $x_{1}, \ldots x_{m}$, abbiamo similmente uno spazio $S_{m}$ a $m$ "dimensioni reali », in cui sono immersi degli altri spazi $S_{0}, \ldots S_{m-1}$ analitici, a $0, \ldots m-\mathbf{I}$ dimensioni reali, in particolare i punti reali $S_{0}$.

I punti di una $V_{r}$, che è data come continuazione analitica di uno $S_{r}(r>0)$, si chiameranno anche punti complessi dello $S_{r}$, e fra essi quelli che non sono reali, non sono situati nello $S_{m}$, si chiameranno punti immaginari dello $S_{r}$, come di solito.

Puó essere che fra i punti della $V_{r}$ (o fra i punti complessi dello $S_{r}$ ) vi siano punti reali, isolati o no, a cui non si possa pervenire col mezzo della detta continuazione analitica, se non pel tramite di punti immaginari. Questi punti (che del resto possono, tutti o in parte, essere sovrapposti a punti dello $S_{r}$ ) li chiameremo punti associati allo $S_{r}$.

Una $V$, si dirà reale, se coincide colla $\bar{V}_{r}$ conjugata, vale a dire, col luogo dei punti complessi-conjugati ai punti della $V_{r}$. Così la $V_{r}$ determinata da uno $S_{r}$ è sempre reale; e in particolare la $V_{m}$ è reale. In tal caso lo $S$, si chiamerd anche un tratto reale della $V_{r}$ reale. Vi possono essere parecchi tratti reali della stessa $V_{r}(0<r<m)$ associati l'uno all'altro. Ma vi sono anche delie varietà reali non godenti di tratti, nemmeno di punti reali. Un ente analitico non reale si dirà immaginario.

I punti di un qualunque ente $S_{r}, V_{r}(r>0)$ o saranno algebrici $\left.{ }^{* *}\right)$, formanti il campo di esistenza dell'ente, oppure punti limiti (trascendenti), formanti, se ve ne sono, il limite naturale dell'ente. Gli uni e gli altri " appartengono all'ente " o "giacciono » o "stanno" sull'ente; ma solamente dei punti algebrici diremo che essi sono compresi o contenuti nell'ente, o che l'ente passa per essi ${ }^{* * *}$ ).

In generale ci basterd considerare i punti generici fra gli algebrici: essi saranno

$\left.{ }^{*}\right)$ Alcune delle nozioni seguenti sono in sostanza identiche a quelle adoperate dall'OsGood (loc. cit.).

**) Nell'intorno d'un punto "algebrico» $n-r$ delle coordinate complesse si comportano come funzioni algebriche delle altre $r$ coordinate. Basta per il nostro scopo il saper tanto. Le molte e difficili questioni intorno ai punti algebrici (e trascendenti) non le toccheremo.

***) Una tale distinzione non è punto superflua. Anmettendo il limite naturale come "contenuto" nell'ente, si sarebbe costretti a dire che un ente analitico puó "contenere» un altro di dimensione eguale, anzi superiore $\left(\eta=\operatorname{sen} \frac{1}{\xi}\right.$, ecc. $)$. 
chiamati punti regolari. Nell'intorno d'un suo punto regolare l'ente si puo sempre rappresentare, in una infiniti di modi, per mezzo di serie di potenze di $r$ parametri essenziali (parametri complessi nel caso delle varietà $V_{r}$, parametri reali nel caso degli spazi $S_{r}$ ) per modo the la matrice funzionale delle coordinate $(\xi \circ x)$ non s'annulla nel punto considerato (centro dello sviluppo). Questi parametri possono senpre scegliersi fra le $m$ coordinate $\xi_{k} \circ x_{k}$, ma non sempre nello stesso modo per tutti $\mathrm{i}$ punti regolari.

Una particolare rappresentazione parametrica, proseguita analiticamente quanto possibile, puó avere un limite naturale sull'ente stesso; essa non dard in generale tutti $\mathrm{i}$ punti dell'ente considerato ${ }^{*}$ ). Scambiando però e riscambiando in modo opportuno i parametri, si puó pervenire a qualsiasi punto.

L'insieme dei punti regolari d'una $V_{r}(r>0)$ è sempre un solo continuo $\left.{ }^{* *}\right)$ compreso nel campo di esistenza: Il campo di regularità della $V_{r}$. L'insieme dei punti regolari di uno $S_{r}$ invece sard̀ formato di uno o più continui, tutti compresi nel campo di esistenza dello $S_{r}$, e separati fra loro da punti algebrici non regolari. Un tale insieme continuo di punti reali lo chiameremo un campo elementare (dello $S_{r}$ ). L'ipocicloide di Steiner, per esempio, uno $S_{1}$ nello $S_{2}$, consta di tre campi elementari e di tre cuspidi, non contenute nei campi.

Un insieme di punti regolari d'un ente analitico (varietà o spazio) puó godere delle proprietà seguenti : a) L'insieme comprende tutti i punti d'un certo intorno d'un qualsivoglia suo punto. $b$ ) L'insieme è continuo, per modo che si puó passare da un qualsiasi suo punto ad un qualsiasi altro mediante continuazione analitica (ed eventualmente scambio dei parametri) pel tramite di punti dell'insieme. c) L'insieme non può ingrandirsi, cioè non è compreso in un altro godente delle proprietà $a$ ), $b$ ), ma esteso fuori del primo. Un tale insieme sarà il campo di regolarità nel caso di una $V_{r}$, ed un campo elementare nel caso d'uno $S_{r}$. Un insieme peró, i cui punti (tutti regolari) riempiano le condizioni $a$ ), $b$ ) (ma eventualmente non la terza), lo chiameremo una regione regolare della $V_{r}$ o dello $S_{r}$. Cosi ogni campo regolare d'una $V_{r}$, ed ogni campo elementare d'uno $S_{r}$, sarà una regione regolare; ma non inversamente.

Un campo elementare lo denoteremo colla lettera $C_{r}$, designando, qui ancora, coll'indice $r$ la dimensione.

$\mathrm{Si}$ vede che una qualsivoglia regione regolare d'uno $S_{r}$, per es. l'intorno d'un

*) Fatto non di rado rimasto inosservato. Naturalmente, in tali casi è facile, ma nowdimeno necessario, di presentare $\mathrm{i}$ teoremi in forma esatta.

Sarà anche opportuno di osservare che a mezzo d'una rappresentazione parametrica l'ente può essere coperto parecchie volte, in tutto o in parte, nello stesso modo come una $V_{\mathrm{t}}$, per es. una retta, puó essere coperta dai differenti rami di una superficie di RiEmanN. In tal caso le singolarità del distendimento, per es. i punti di diramazione, non saranno sempre singolarità dell'ente stesso.

**) Secondo il Cantor un "continuo è sempre chiuso. Noi non possiamo accettare una tale restrizione. Si vegga la "Geometrie der Dynamen " dell'autore (Lipsia, 1903), a piè della pagina 248. 
suo punto regolare, determina completamente: $1^{\circ}$ Un campo elementare $C_{r} ; 2^{\circ}$ lo spazio $S_{r}$, su cui il campo $C_{r}$ è situato; $3^{\circ}$ tutti gli altri campi elementari $C_{r}^{\prime}, C_{r}^{\prime \prime}, \ldots$ dello stesso spazio (se ve ne sono); $4^{\circ}$ la varietà $V_{r}$, su cui lo $S_{r}$ è situato; $5^{\circ}$ tutti i punti, campi, spazi reali, associati allo $S_{r}(r>0)$.

Se diciamo che un ente analitico s'appoggia su di un altro o che questo porta il primo, vogliamo esprimere che almeno UNA REGIONE REGOLARE del secondo ì compresa nel. CAMPO DI ESISTENZA del primo.

Cosi, ogni ente analitico "s'appoggia " su sè stesso, e su tutti i suoi punti algebrici. Uno $S_{r}$ definente una $V_{r}$ reale porta questa. La $V_{m}$ si appoggia su tutte le $V_{r}$ e su tutti gli $S_{r .}-$ Si potrebbe credere che la nostra definizione fosse superflua nel caso in cui i due enti siano varietà (cioè, enti complessi): si potrebbe pensare che tutta la varietà portante dovesse giacere sulla varietì portata. Ma una tale conclusione sarebbe sbagliata. Ecco un esempio *).

Sia $f(\xi)$ una funzione analitica regolare nell'interno del cerchio $|\xi|<\mathrm{I}$, ma avente questo cerchio come limite naturale. Nella $V_{;}(\xi, n, \zeta)$ la $V_{2}: \zeta-n f(\zeta)=0$ s'appoggia sulla $V_{1}: n=0, \zeta=0$, comprendendone tutti i punti situati nell'interno del cerchio assunto, i cui punti stessi giacciono ancora sulla $V_{2}$. Ma non si potrebbe dire che anche $\mathrm{i}$ punti esterni al cerchio soddisfino alla stessa equazione, perchè per essi l'espressione $f(\zeta)$ è priva di senso. Dunque questi punti non appartengono alla $V_{2}$.

$\mathrm{Si}$ vede di qui che la varietà portante puó essere tagliata in parti dal limite naturale della varietà portata, anche nel mezzo d'un suo campo regolare.

Se la varieta portata è algebrica, una tale circostanza è impossibile. Ma nel caso degli spazi già gli enti algebrici offrono, come è ben noto, delle proprietà simili.

Se tutti i punti dell'ente portante appartengono all'ente portato, abbiamo fra loro una connessione più intima: Diremo che l'ente portante è contenuto o compreso nell'ente portato, o che questo passa per l'ente portante. Cosi ogni ente è contenuto in sè stesso e passa per sè stesso. Nel nostro esempio il cerchio è contenuto nella $V_{\mathrm{I}}$, ma non nella $V_{2}$, giacchè tutti i suoi punti sono punti limiti della $V_{2}$.

E chiaro che la differenza tra una varieta $V_{r}$ ed uno spazio $S_{2 r}$ di dimensione pari non è essenziale. Le due parole designano piuttosto due modi di riguardare la stessa cosa. La varietà si potrà trattare come uno spazio. Possiamo scindere le variabili complesse nelle loro componenti reali : avremo uno spazio $S_{2 r}$. Possiamo estendere queste componenti al campo complesso: avremo definito una $V_{2 r}$; e cosi via, senza fine. E appunto questo, che vogliamo fare ora, fermandoci peró dopo il primo passo.

Tratteremo un campo di $n$ variabili complesse

$$
z_{1}=x_{1}+i y_{1}, \ldots z_{n}=x_{n}+i y_{n}
$$

come uno spazio $S_{2 n}$ di $2 n$ dimensioni reali, e come immerso in una varietà $V_{2 n} d i$

*) Un altro esempio si trova nell'articolo citato dell'Osgood ( $\mathrm{n}^{\circ} 47$ del testo). 
$2 n$ dimensioni complesse, le cui variabili (complesse) saranno

$$
\xi_{1}, n_{1} ; \ldots \xi_{n}, n_{n} \text {. }
$$

Introdurremo poi un ulteriore termine tecnico, per distinguere dagli altri quegli spazi $S_{2 \rho}$ dello $S_{2 n}\left(x_{1}, y_{\mathrm{I}} ; \ldots x_{n}, y_{n}\right)$ che sono varietd $V_{\rho}^{\prime}$ nella $V_{n}^{\prime}\left(z_{1}, \ldots z_{n}\right)$, e le loro continuazioni analitiche $V_{20}$ nella $V_{2 n}\left(\xi_{1}, n_{1} ; \ldots \xi_{n}, n_{n}\right)$ dal resto delle varietd analitiche a $2 \rho$ dimensioni complesse. Li cbiameremo spazi e varietà sinettici (includendo in tale espressione gli enti $S_{2 n}$ e $V_{2 n}$ stessi, e tutti i punti $S_{o}$ reali). Cosi uno spazio sinettico nello $S_{2 n}$ non sarà altro che una varieta analitica in una $V_{n}^{\prime}$. Le varietà sinettiche peró saranno varietà analitiche molto speciali; cioè quelle che possono riguardarsi come continuazioni analitiche (nella $V_{2 n}$ ) degli spazi sinettici, che gia sono

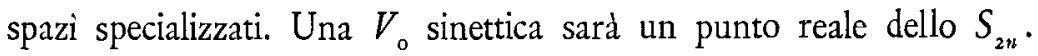

Interpretando, per esempio, le variabili $x_{1}, y_{1}, x_{2}, y_{2}$ come coordinate ortogonali cartesiane in uno spazio euclideo $S_{4}$ (reale) a quattro dimensioni, avremo da considerare: Degli spazi $S_{0}$, o punti reali; degli spazi $S_{1}$, o "TRATTI REALI di curve analitiche"; degli spazi $S_{2}$, o "TRATTI REALI di superficie analitiche "; e cosi degli spazi $S_{3}$ e lo spazio $S_{4}$ stesso. Fra questi saranno sinettici: $1^{\circ}$ Gli $S_{0} \cdot 2^{\circ}$ Quegli spazi $S_{2}$, che si possono rappresentare (in una regione regolare) a mezzo d'un parametro complesso $t$ nella forma " sinettica »

\section{$3^{0}$ Lo spazio $S_{4}$.}

$$
z_{1}=f_{1}(t), \quad z_{2}=f_{2}(t)
$$

Nella varietà euclidea $V_{4}$ invece consideriamo delle varietà $V_{0}, V_{\mathrm{I}}, V_{2}, V_{3}\left(\mathrm{e} V_{4}\right)$, luoghi analitici di $\infty^{0}, \infty^{2}, \infty^{4}, \infty^{6}$ (e $\left.\infty^{8}\right)$ punti complessi, e specialmente le $V_{\mathrm{r}}$ e $V_{2}$, che sono state chiamate "curve » e "superficie» analitiche ${ }^{*}$ ). Fra le reali ci sono le varieta sinettiche: Le $V_{\mathrm{o}}$ reali, identiche agli $S_{0}$, cioè ai punti reali; poi quelle $V_{2}$ che derivano dagli $S_{2}$ sinettici; ed in fine la $V_{4}$.

Posto $t=\varphi+i \psi$, scriviamo cosi le due equazioni precedenti:

$$
\begin{aligned}
& x_{1}+i y_{1}=f_{1}(\varphi+i \psi), \\
& x_{2}+i y_{2}=f_{2}(\varphi+i \psi) .
\end{aligned}
$$

*) L'uso delle parole curva e superficie oscilla in guisa da non farsene un'idea. In tanti casi non si sa che cosa voglia dire un autore. Il nostro uso coincide con quello più abituale nella geometria projettiva. Ma riguardo allo stato attuale della letteratura sarà opportuno osservare che una curva algebrica non è analitica (secondo le solite definizioni di queste parole), se non è irreducibile, e che non si devono confondere i sei concetti: Curva (analitica oppure atgebrica) reale; curva dotata di tratti reali; l'insieme dei punti reali di una curva reale (se ve ne sono); l'insieme dei tratti reali di una curva reale (se ve ne sono); singolo tratto reale di una tale curva; regione regolare d'un tratto reale. Denotare tutti questi concetti, e non so quanti altri, colla stessa parola, senza le debite definizioni e senza coerenza è un adattarsi all'equivoco. Eे questo un fatto, che caratterizza la condizione presente d'una gran parte della letteratura geometrica. Il voler chiudere gli occhi di fronte ad una tale confusione, il preferire una semplicita apparente alla vera semplicità, che consiste nel definire esattamente e nel limitare propriamente tutti $\mathrm{i}$ concetti usati, ecco uno dei più grandi ostacoli per il progresso della geometria. Si veda la prefazione del libro citato "Geometrie der Dynamen». 
$\mathrm{Da}$ queste derivano quattro equazioni reali :

$$
\begin{aligned}
& x_{1}=\varphi_{1}(\varphi, \psi), \quad y_{1}=\psi_{1}(\varphi, \psi), \\
& x_{2}=\varphi_{2}(\varphi, \psi), \quad y_{2}=\psi_{2}(\varphi, \psi) .
\end{aligned}
$$

Continuando ora i parametri $\phi, \psi$ nel campo complesso, cioè sostituendoli con valori $u, v$ complessi abbastanza vicini, avremo nelle formule

$$
\begin{aligned}
& \xi_{1}=\varphi_{1}(u, v), \quad n_{1}=\psi_{1}(u, v), \\
& \xi_{2}=\varphi_{2}(u, v), \quad n_{2}=\psi_{2}(u, v)
\end{aligned}
$$

la rappresentazione analitica della varietà $V_{2}$ sinettica più generale, in una sua regione regolare. $\mathrm{E}$ cosi in casi superiori.

Supponiamo ora che uno $S_{2 \beta}$ sia dato per mezzo di $2 \xi$ parametri reali essenziali, in una sua regione regolare. Come potremo riconoscere se questo $S_{2 \beta}$ sia sinettico? $E$ trovatolo sinettico: Come potremo rappresentarlo per mez̃zo di $\rho$ parametri complessi? La risposta è pressochè immediata. Se una rappresentazione sinettica già fosse stata trovata, le componenti reali dei $\rho$ parametri complessi potrebbero sostituirsi invece dei $2 \rho$ parametri dati, ed inversamente. Ma sostituendo le dette componenti con parametri nuovi complessi, nel modo più generale, non avremo fatto altro che introdurre dei parametri sovrabbondanti. Quindi, proseguendo i parametri dati nel campo complesso, non usciremo fuori dello spazio dato $S_{2 p}$.

I. (Teorema fondamentale). Un dato $S_{2 \rho} \dot{e}$ sinettico, se, sostituendo $i$ suoi $2 \rho$ parametri reali con valori vicini complessi, nelle espressioni trovate

$$
z_{k}=f_{k}\left(t_{1}, \ldots t_{2 \rho}\right)
$$

appunto $p$ parametri sono sovrabbondanti.

Rimovendo quei parametri, si ba una rappresentazione sinettica dello $S_{2 \rho}$, od almeno d'una sua regione regolare, per mezzo di p parametri complessi.

Teoricamente, il rimuovere quei parametri, che fossero sovrabbondanti, non offre alcuna difficoltà; basta sostituirli con funzioni (scelte in modo proprio) degli altri parametri, per es. con valori costanti.

Ma lo stesso ragionamento ci conduce subito ad un teorema più generale:

II. Per ogni spazio $S_{r}$ passa uno spazio $S_{2 \beta}$ sinettico ben determinato $\left.{ }^{*}\right)$. $S u$ di questo s'appoggiano tutti gli spazî portati dallo $S_{r}$.

Per trovar lo $S_{2 p}$, basta proseguire gli $r$ parametri reali dello $S_{r}$ nel vicino campo complesso, e rimuovere quegli $r-p$ parametri complessi, che risultassero eventualmente sovrabbondanti. Si vede che si ha sempre

$$
r \leq 2 p \leq 2 r, \quad p \leq n
$$

*) Cfr. SEgRe, loc. cit., pag. 440. 
e si trovano anche subito i criteri per tutti i casi, a mezzo di determinanti funzionali (Teorema I). Si vede che, dovunque lo $S_{r}$ ha un punto regolare (o algebrico), lo $S_{2 p}$ ha anche un punto regolare (o algebrico).

Potremmo gia trarre parecchie conseguenze dal Teorema II. Ma prima di far ciò, ci pare opportuno istituire alcune considerazioni d'un carattere differente, le quali ci condurranno a qualche generalizzazione del Teorema II. (Veggansi i Teoremi VII, XIII).

Si prova subito:

III. Vi sono due classi distinte di trasformazioni puntuali reali (e di necessità analitiche) godenti della proprietà di permutar fra loro gli spazi sinettici:

Le "trasformazioni sinettiche "

$$
Z_{k}=F_{k}\left(z_{\mathrm{I}}, \ldots z_{n}\right)
$$

e le "trasformazioni antisinettiche "

$$
\bar{Z}_{k}=F_{k}\left(z_{i} \ldots z_{n}\right)
$$

Nelle trasformazioni della prima classe le variabili trasformate $Z_{k}$ sono funzioni analitiche aventi un campo comune di esistenza, del resto arbitrarie, colla sola restrizione di essere indipendenti. Nel secondo caso i valori conjugati

delle variabili complesse

$$
\bar{Z}_{k}=X_{k}-i Y_{k}
$$

$$
Z_{k}=X_{k}+i Y_{k}
$$

sono espressi nello stesso modo.

E naturalmente sottinteso che si parla solo di quegli spazi che entrano, ed in quanto entrano, nel campo di regularità di una tale trasformazione (campo continuo ed unico, in cui il determinante funzionale della trasformazione non s'annulla).

In quanto sia possibile di eseguire l'una dopo l'altra due tali trasformazioni della prima specie (per es.), il prodotto loro sard una trasformazione dello stesso tipo. Abbiamo cosi un "gruppo continuo infinito ", e nell'insieme delle due classi un altro gruppo infinito detto " misto ». Sard opportuno di chiamare questi due gruppi riproduttivi dell'insieme degli spazi sinettici “ il gruppo sinettico» ed " il gruppo sinettico ampliato ${ }^{*}$ ). Interpretando le variabili $x_{k}, y_{k}$ e $X_{k}, Y_{k}$ come coordinate cartesiane ortogonali nello spazio euclideo $S_{2 n}$, vediamo che entrambe le classi di trasformazioni sono conformi per le regioni regolari a due dimensioni (in quanto entrano nel campo di regolarita della trasformazione considerata).

Continuiamo ora le trasformazioni definite nel campo complesso, o nella $V_{2 n}$, il che può farsi senz'altro. Omettendo gl'indici, troviamo nel prino caso:

*) Una parte essenziale delle proprietà qui rilevate di questo gruppo importante è già stata sviluppata nella Memoria citata del SEgre. Veggansi specialmente i numeri 18, 26, 27. 
e nel secondo:

$$
\begin{aligned}
& \boldsymbol{\Xi}=\frac{\mathrm{I}}{2}[F(\xi+i n)+\bar{F}(\xi-i n)], \\
& \mathbf{H}=\frac{\mathrm{I}}{2 i}[F(\xi+i n)-\bar{F}(\xi-i n)],
\end{aligned}
$$

$$
\begin{aligned}
& \Xi=\frac{\mathrm{I}}{2}[F(\xi+i n)+\bar{F}(\xi-i n)], \\
& \mathbf{H}=-\frac{\mathrm{I}}{2 i}[F(\xi+i \eta)-\bar{F}(\xi-i n)] .
\end{aligned}
$$

$\mathrm{Da}$ queste formule deriva subito il teorema:

IV. Rispetto al gruppo sinettico ampliato $i$ punti $(\xi, n)$ della varietà $V_{2 n}$, cioè $i$ punti detti "complessi dello spazio $S_{2 n}$ ", stanno in relazione invariabile ed univoca colle coppie ben ordinate $\left(z^{\prime} ; z^{\prime \prime}\right)$ di punti reali dello stesso spazio:

$$
\begin{aligned}
& \xi-i n=x^{\prime}-i y^{\prime}=\overline{z^{\prime}}, \\
& \xi+i n=x^{\prime \prime}+i y^{\prime \prime}=z^{\prime \prime} .
\end{aligned}
$$

Cosi ogni punto complesso $(\xi, n)$ ba due immagini reali

$$
z^{\prime}=x^{\prime}+i y^{\prime}, \quad z^{\prime \prime}=x^{\prime \prime}+i y^{\prime \prime},
$$

che sono scambiate tra di loro, se si passa dal punto $(\bar{\zeta}, n)$ al punto gemello $\left(\bar{\xi}, \bar{\gamma}_{1}\right)$.

Naturalmente i punti $z^{\prime}, z^{\prime \prime}$ coincidono, se il punto $(\xi, n)$ diventa reale, e viceversa.

Si domanda ora: quali sono le relazioni geometriche fra i quattro punti $(\xi, n)$, $(\bar{\xi}, \bar{n}),\left(x^{\prime}, y^{\prime}\right),\left(x^{\prime \prime}, y^{\prime \prime}\right)$ ? La risposta è evidente:

V. Vi sono nella varietà $V_{2 n}$ [euclidea] due schiere (gemelle) di varietà [piane parallele], entrambe dipendenti da $n$ parametri complessi $\overline{z_{k}^{\prime}}, \tilde{z}_{k}^{\prime \prime}:$ le PRotovarieta ${ }^{*}$ )

$$
\begin{aligned}
& V_{n}^{\prime}: \quad \check{\zeta}_{k}-i n_{k}=\bar{z}_{k}^{\prime}=\text { cost. } \quad(k=\mathrm{I}, \ldots n) \\
& V_{n}^{\prime \prime}: \quad \xi_{k}+i n_{k}=z_{k}^{\prime \prime}=\text { cost. } \quad(k=\mathrm{I}, \ldots n) \text {. }
\end{aligned}
$$

Le trasformazioni sinettiche permutano fra loro le varietà di ciascuna schiera, le une nel modo analitico piu generale, le altre nel modo gemello (cioè nel modo complessoconjugato):

$$
\begin{aligned}
& \overline{Z^{\prime}}=\bar{F}\left(\overline{z^{\prime}}\right), \\
& Z^{\prime \prime}=F\left(z^{\prime \prime}\right) .
\end{aligned}
$$

Le trasformazioni antisinettiche invece mutano l'una schiera nell'altra:

$$
\begin{aligned}
& Z^{\prime \prime}=\bar{F}\left(\overline{z^{\prime}}\right), \\
& \overline{Z^{\prime}}=F\left(z^{\prime \prime}\right) .
\end{aligned}
$$

*) Nozione introdotta dal SEGRE, come anche il termine «figure gemelle ». 
Le due immagini $z^{\prime}, z^{\prime \prime}$ d'un punto complesso sono, secondo l'ordine, $i$ (soli) punti reali delle due protovarietà passanti per il punto complesso, oppure, nell'ordine inverso, $i$ punti reali delle protovarietà passanti per il punto gemello.

S'intende che il gruppo sinettico ampliato è caratterizzato anche da una di queste proprietd IV, V.

Dal punto di vista a cui ora siamo arrivati, si possono studiare, fra altri luoghi, tutte le varietà analitiche comprese nella $V_{2 n}$. Introduciamo per un momento una varietà ausiliare $V_{2 n}^{*}$ (la quale non differisce del resto dalla $V_{2 n}$ se non per la scelta delle coordinate) interpretando i sistemi delle $2 n$ variabili complesse

$$
\bar{z}_{1}^{\prime}, \ldots \overline{z_{n}^{\prime}} ; \quad z_{\mathrm{I}}^{\prime \prime}, \ldots \bar{z}_{n}^{\prime \prime}
$$

come punti della $V_{2 n}^{*}$. Ad ogni $V_{r}$ corrisponderd una $V_{r}^{*}$ e viceversa. Quindi nello spazio $S_{2 n}$ avremo due spazi sinettici $\bar{S}_{2 \rho^{\prime}}^{\prime}, S_{2 \rho^{\prime \prime}}^{\prime \prime}$ luoghi dei punti reali $\bar{z}^{\prime}, z^{\prime \prime}, \mathrm{i}$ quali, se $\rho^{\prime}+\rho^{\prime \prime}>r$, saranno legati da

$$
p^{\prime}+p^{\prime \prime}-r
$$

vincoli analitici, o meglio sinettici $\Phi\left(\overline{z^{\prime}}, z^{\prime \prime}\right)=0$ indipendenti. Mutando i punti $\overline{z^{\prime}}$ nei punti $z^{\prime}$, che loro corrispondono nella trasformazione reale

$$
X_{k}-i Y_{k}=x_{k}+i y_{k}
$$

detta conjugio dal SEGRE, otterremo ancora due spazî sinettici, $S_{2 \rho^{\prime}}^{\prime}$ e $S_{2 \rho^{\prime \prime}}^{\prime \prime}$ :

VI. Le immagini reali dei punti complessi $(\xi, n)$ d'una qualsiasi $V_{r}$, sono $i$ punti $z^{\prime}$ e $z^{\prime \prime}$ di due spazi $S_{2 \rho^{\prime}}^{\prime}$ e $S_{2 \rho^{\prime \prime}}^{\prime \prime}$ SInetTici. Vi sono fra loro $\rho^{\prime}+p^{\prime \prime}-r$ legami antrSINETTICI indipendenti ${ }^{*}$ ).

Viceversa, ogni ente analitico costituito da tali coppie di punti rappresenterà una $V_{\text {r }}$ analitica.

Cosi, esprimendo le $2 n$ variabili $\overline{z^{\prime}}, z^{\prime \prime}$ nel modo analitico più generale per mezzo di $r$ parametri complessi essenziali, otteniamo tutte le $V_{r}$, o regioni di esse.

Vi sono due famiglie notevoli di $V_{r}$ immaginarie, cioè quelle, in cui l'uno dei punti $z^{\prime}, z^{\prime \prime}$ rimane fisso, mentre l'altro percorre un qualsivoglia $S_{2 \rho}$ sinettico. Le chiameremo varietà caratteristiche, includendo in questo nome tutti i punti complessi. Si vede subito che una varietd caratteristica o sard una protovarieta $(\rho=n)$, oppure sarà immersa in una protovarieta $(\rho<n$; in due, se $\hat{\rho}=0)$. Sostituendo il punto fisso con un altro, si trova una nuova varietà caratteristica, derivata dalla prima per mezzo d'una traslazione. Da ció si deduce che tutte le varietá, per cui $\rho^{\prime}+\rho^{\prime \prime}-r=0$, $\rho^{\prime}>0, \rho^{\prime \prime}>0$, sono varietà di traslazione o di scorrimento, secondo il senso abituale di queste parole.

Se la $V_{r}$ è reale, si puó passare dalla coppia generica $\left(z^{\prime}, z^{\prime \prime}\right)$ alla coppia inversa enti sinettici. 
$\left(z^{\prime \prime}, z^{\prime}\right)$ per mezzo di continuazione analitica reale. Quindi si ha $\rho^{\prime}=\rho^{\prime \prime}$; e di più gli $S_{2 p^{\prime}}^{\prime}, S_{2 p^{\prime \prime}}^{\prime \prime}$ sinettici devono coincidere. Dunque:

VII. Una qualunque $V_{r}$ REALE è contenuta in una $V_{2 p}$ sinettica ben determinata.

Tutte le varietà sinettiche appoggiate sulla $V_{r}$ s'appoggiano anche su questa $V_{2 f}$.

Si vede che si ha sempre, anche qui:

$$
r \leq 2 p \leq 2 r, \quad p \leq n .
$$

Non sarebbe difficile lo studiare tutti $i$ casi; ma noi vogliamo restringerci ai casi più semplici ed interessanti, vale a dire ai casi estremi $2 \rho=r$ e $\rho=r$.

Se $r=2 \rho$, gid la $V$, stessa è sinettica. Ne deriva una conseguenza importante:

VIII. Gli spazî $S_{2 p}$ sinettici sono appunto quelli, le cui coppie di punti sono le immagini reali dei loro punti complessi.

Cosi, prendendo una qualsivoglia coppia $z^{\prime}, z^{\prime \prime}$ di punti reali (regolari, algebrici o generali) d'un tale spazio, avremo le immagini d'un qualsivoglia punto $(\xi, n)$ (regolare, algebrico o generale) della $V_{2 \rho}$ sinettica, che viene determinata dallo spazio $S_{2 \rho}$.

Alla stessa conclusione arriviamo per altre vie.

E chiaro che ogni $S_{2 \rho}$ equivale nel gruppo sinettico allo spazio speciale

$$
z_{p+\mathbf{r}}=0, \ldots z_{n}=0 .
$$

Ma evidentemente questo spazio contiene le immagini di tutti i suoi punti immaginari. Di qui si deduce che il Teorema VIII è vero per quei punti immaginari dello $S_{2 p}$, che sono regolari ed abbastanza vicini ai punti reali. Ma questo è sufficiente per dedurne il teorema in tutta la sua generalità. Basta applicare la continuazione analitica.

Finalmente si puó stabilire il teorema mediante verifica diretta. Sia $z=f(t)$ una rappresentazione sinettica dello $S_{2 \rho}$. Posto $t=\sigma+i \tau$, avremo nelle formule

$$
\begin{aligned}
& \xi=\frac{\mathrm{I}}{2}[f(\sigma+i \tau)+\bar{f}(\sigma-i \tau)], \\
& n=\frac{\mathrm{I}}{2 i}[f(\sigma+i \tau)-\bar{f}(\sigma-i \tau)]
\end{aligned}
$$

la continuazione analitica dello $S_{2 \rho}$ nel campo complesso $V_{2 n}$. Esse equivalgono a $\xi+i n=z=f(t)$, se i parametri $\sigma, \tau$ divengono reali. Lasciandoli complessi, $\mathrm{i}$ punti

$$
\begin{aligned}
& z^{\prime}=\bar{\xi}+i \bar{n}=f(\bar{\sigma}+i \bar{\tau}), \\
& z^{\prime \prime}=\xi+i n=f(\sigma+i \tau)
\end{aligned}
$$

saranno entrambi situati sullo $S_{2 \rho}$, se pure le funzioni $f_{k}$ esistono per tali argomenti (condizione necessaria per l'esistenza delle funzioni $\xi$, $n$, i cui argomenti $\sigma+i \tau \mathrm{e}$ $\sigma-i \tau$ sono variabili indipendenti). Quindi si può scrivere:

$$
\begin{aligned}
& \xi-i n=\overline{z^{\prime}}=x^{\prime}-i y^{\prime}, \\
& \xi+i n=z^{\prime \prime}=x^{\prime \prime}+i y^{\prime \prime} .
\end{aligned}
$$


Ecco ora una conseguenza notevole:

IX. Tutte le varietà $V_{2 \rho}$ sinettiche sono unicursali, cioè, ciascuna contiene un solo tratto reale $S_{2 \rho}$, privo di punti associati. (Veggasi più sopra).

Un po' più generale ancora è il teorema seguente, che deriva dagli stessi principî:

$\mathrm{X}$. Una qualunque varietà portante una data varietà $V_{2 p}$ sinettica non può uscire fuori di questa, se non è contenuta almeno in una $V_{2 p-2}$ sinettica. Quindi: Tutti $i$ punti reali associati ad un $S_{r}$ appartengono allo spazio $S_{2 \rho}$ sinettico individuato dallo $S_{r}$.

Finalmente osserviamo (per $p>0$ ):

Tutte le varietà $V_{2 \rho}$ sinettiche sono varietà di scorrimento.

Ciascuna si puó generare in due modi per mezzo d'una traslazione a $\rho$ parametri complessi, il cui oggetto è l'una o l'altra di due varieta caratteristiche $V_{\rho}^{\prime}$ e $V_{\rho}^{\prime \prime}$.

Inversamente, ogni varietà REALE generata in tal modo sarà sinettica: Essa coincide (se $\rho>0$ ) colla continuazione analitica d'uno spazio $S_{2 p}$ sinettico. Vedremo ora come queste osservazioni si possano generalizzare.

Il Teorema VIII dice (fra l'altro) che i punti complessi $(\xi, n)$ e $(\bar{\xi}, \bar{n})$ rappresentati dalle formule

$$
\begin{array}{ll}
2 \bar{\zeta}=\left(x^{\prime \prime}+x^{\prime}\right)+i\left(y^{\prime \prime}-y^{\prime}\right), & 2 i \text { in }=\left(x^{\prime \prime}-x^{\prime}\right)+i\left(y^{\prime \prime}+y^{\prime}\right), \\
2 \bar{\xi}=\left(x^{\prime}+x^{\prime \prime}\right)+i\left(y^{\prime}-y^{\prime \prime}\right), & 2 i \bar{n}=\left(x^{\prime}-x^{\prime \prime}\right)+i\left(y^{\prime}+y^{\prime \prime}\right)
\end{array}
$$

stanno sulla $V_{2 \rho}$, se i punti $\left(x^{\prime}, y^{\prime}\right),\left(x^{\prime \prime}, y^{\prime \prime}\right)$ dello $S_{2 \rho}$ sinettico son reali. Basta enunciare il nostro risultato in tale forma, per vedere che l'ultima restrizione è superflua. Solamente, sostituendo le variabili reali $x^{\prime}, y^{\prime}, x^{\prime \prime}, y^{\prime \prime}$ con valori complessi $\xi^{\prime}, n^{\prime}$, $\xi^{\prime \prime}, n^{\prime \prime}$, non otterremo più punti gemelli, ma invece una coppia $\left(\xi_{1}, n_{1}\right),\left(\xi_{2}, n_{2}\right)$ generica di punti della $V_{2 \rho}$, data dalle equazioni involutorie:

$$
\begin{aligned}
& \xi_{1}-i \eta_{1}=\xi^{\prime}-i \eta^{\prime}, \quad \xi_{1}+i \eta_{1}=\xi^{\prime \prime}+i \eta^{\prime \prime}, \\
& \xi_{2}-i \eta_{2}=\xi^{\prime \prime}-i \eta^{\prime \prime}, \quad \xi_{2}+i \eta_{2}=\xi^{\prime}+i n^{\prime} .
\end{aligned}
$$

L'interpretazione è immediata:

XI. Assunti due qualsivogliano punti d'una varietà sinettica, anche $i$ due altri punti di riscontro delle protovarietà passanti per essi stanno sulla varietà ${ }^{*}$ ).

La stessa conseguenza si trae del resto dalla considerazione della varietd speciale $\xi_{\rho+1}=0, n_{\rho+1}=0, \ldots \xi_{n}=0, n_{n}=0$; ed anche qui si trovano subito i parametri rappresentativi dei quattro punti considerati. Basta sostituire nelle formule

$$
\begin{aligned}
& \xi=\frac{\mathrm{I}}{2}[f(\sigma+i \tau)+\bar{f}(\sigma-i \tau)], \\
& n=\frac{\mathrm{I}}{2 i}[f(\sigma+i \tau)-\bar{f}(\sigma-i \tau)]
\end{aligned}
$$

valori complessi $\sigma, \tau$ connessi dalle stesse equazioni:

*) SEGRE, loc. cit., pag. 455 . 


$$
\begin{aligned}
& \sigma_{1}-i \tau_{1}=\sigma^{\prime}-i \tau^{\prime}, \quad \sigma_{1}+i \tau_{1}=\sigma^{\prime \prime}+i \tau^{\prime \prime} \\
& \sigma_{2}-i \tau_{2}=\sigma^{\prime \prime}-i \tau^{\prime \prime}, \quad \sigma_{2}+i \tau_{2}=\sigma^{\prime}+i \tau^{\prime} ;
\end{aligned}
$$

come si verifica direttamente.

Mentre il Teorema VIII caratterizza gli spazi sinettici, la proprieti enunciata nel Teorema XI definisce una famiglia più estesa di varietà, le quali vogliamo chiamare varietà di SEgre, includendo anche in questo nome la $V_{2 n}$ e tutti i punti complessi.

$\dot{\mathrm{E}}$ cosa facile trovare tutte le varietd di SEGRE. Sia $\left(\zeta_{1}, r_{1}\right)$ un punto regolare fisso, $\left(\zeta_{2}, n_{2}\right)$ un punto variabile di una tale $V_{r}$. Si vede che i punti $\left(\zeta^{\prime}, r_{1}^{\prime}\right)$ e $\left(\zeta^{\prime \prime}, n^{\prime \prime}\right)$ stanno su due varietí caratteristiche $V_{r^{\prime}}^{\prime}, V_{r^{\prime \prime}}^{\prime \prime}$, intersezioni della $V_{r}$ colle due protovarietà $V_{n}^{\prime}, V_{n}^{\prime \prime}$ passanti per il punto $\left(\zeta_{1}, n_{1}\right)$. Ma sulle $V_{r^{\prime}}^{\prime}, V_{r^{\prime \prime}}^{\prime \prime}$ i punti $\left(\zeta^{\prime}, n^{\prime}\right)$ e $\left(\xi^{\prime \prime}, n^{\prime \prime}\right)$ possono scegliersi arbitrariamente, determinando cosi il punto $\left(\xi_{2}, n_{2}\right)$. Quindi si ha $r^{\prime}+r^{\prime \prime}=r$. Le due $V_{, \prime}^{\prime}, V_{r^{\prime \prime}}^{\prime \prime}$, che anzi si possono prefissare, determinano la $V_{r}$, che sarà rappresentata da formule del tipo

$$
\xi+i n=\varphi(s), \quad \xi-i n=\bar{\psi}(t) \text {. }
$$

Abbiamo cosi trovato di nuovo le varietà di scorrimento già considerate di passaggio. Naturalmente peró i due sistemi di funzioni $\varphi(s)$ e $\bar{\psi}(t)$ possono ridursi a sistemi di valori costanti: sarà opportuno includere anche questi casi nel concetto delle "varietà di scorrimento ", chiamandoli varietà di scorrimento improprie. Cosi tutte le varietà caratteristiche (e fra loro tutti i punti complessi) daranno tutte le varieta di scorrimento improprie.

XII. Le varietà di SEgRE sono quelle, le cui immagini reali sono costituite da TUTTE le coppie $z^{\prime}$, $z^{\prime \prime}$ di punti reali di due spazi sinettici $S_{2 r^{\prime}}^{\prime}$ e $S_{2 r^{\prime \prime}}^{\prime \prime}$ :

$$
z_{i}^{\prime}=\psi_{i}\left(\bar{t}_{\mathrm{s}} \ldots \bar{t}_{\mathrm{r}^{\prime}}\right), \quad z_{i}^{\prime \prime}=\varphi_{i}\left(s_{s} \ldots s_{r^{\prime \prime}}\right) \text {. }
$$

Nella varietì euclidea $V_{2 n}$ esse coincidono colle varietà di scorrimento, che sono generate in due modi per mezzo di varietà caratteristiche ${ }^{*}$ ).

Ora vediamo che il Teorema VII, che gid era una generalizzazione del Teorema II, puó generalizzarsi ancora come segue:

XIII. Una qualunque $V_{r}$ è contenuta in una varietà di SEGRE ben determinata.

$\mathrm{Su}$ di questa s'appoggiano tutte le varietà di $\mathrm{SEGRE}$ appoggiate sulla $V_{r}$.

E chiaro che tutta la teoria delle varieta sinettiche puo estendersi alle varietà di SEgre, la cui dimensione complessa non è ristretta ai numeri pari. La dimensione complessa della varietà di SEgRE individuata da una $V_{r}$ non puó superare il numero $2 r$; si possono definire trasformazioni complesse [pit generali delle trasformazioni (reali) sinettiche ed antisinettiche] che permutano fra loro le varieta di SEgre; e via discorrendo. Noi non vogliamo trattenerci su queste generalizzazioni; ci bastera rilevare un'osservazione evidente:

*) Negli accennati casi speciali, l'uno degli scorrimenti (a $r^{\prime \prime}$ e $r^{\prime}$ parametri complessi) o ambedue si riducono all'identità. 
XIV. Le varietà reali di SEgRE suno le varietà sinettiche.

Ecco un complemento essenziale del Teorema IX: Si potrebbe pensare che la nostra definizione delle varietà sinettiche fosse troppo ristretta; che ci fossero delle altre varietà reali, godenti, nel campo complesso, delle stesse proprietà, tranne l'esser dotate di tratti reali. Tali varietà non esistono.

Ritornando ora sul Teorema VII, consideriamo il secondo caso estremo, supponendo $p=r, r \leqslant n$ (ed in primo luogo $r=n$ ). Una $V_{r}$ reale od uno spazio $S_{r}$ od un campo elementare $C_{r}$, ecc., per cui $\rho=r$, li chiameremo ordinari (e tutti gli altri straordinari). Così tutti gli $S_{1}$ e tutti gli $S_{2}$ non sinettici sono ordinari. Uno $S_{n}$ è ordinario, se non v'è vincolo sinettico $F\left(\tilde{z}_{1}, \ldots z_{n}\right)=0$ fra le coordinate complesse dei suoi punti, ecc. Lo spazio $y_{\mathrm{s}}=0, \ldots y_{n}=o$, per es., è ordinario.

Rileviamo da prima il teorema:

XV. Se due regioni ordinarie, parti di campi $C_{n}, C_{n}^{\prime}$ ORDINARI nello spazio $S_{2 n}$, sono riferite univocamente l'una all'altra per mezzo di una corrispondenza analitica arbitraria (il che può sempre farsi esprimendole tutt'e due mediante gli stessi parametri $\sigma_{1}, \ldots \sigma_{n}$ ), esiste sempre una sola trasformazione sinettica ed una sola trasformazione antisinettica, le quali valgono a stabilire la data corrispondenza fra le due regioni.

Questo è una semplice applicazione del Teorema II. Nel primo caso basta sostituire le $n$ variabili complesse $z_{k}$ colle $2 n$ variabili complesse $z_{k}$, $z_{k}^{\prime}$; nel secondo caso si procede in un modo quasi identico.

Confrontando le due asserzioni del Teorema XV, troviamo:

XVI. Ogni campo $C_{n}$ ORDINARIO determina in un suo intorno una "riflessione antisinettica ", vale a dire, una trasformazione antisinettica (di necessità involutoria) riproduttiva di tutti $i$ punti del $C_{n}$.

Naturalmente questa riflessione è collegata al $C_{n}$ in modo invariabile rispetto al gruppo sinettico ampliato. Per trovarla, basta qui ancora sostituire nelle formule

$$
z_{k}=x_{k}\left(\sigma_{1}, \ldots \sigma_{n}\right)+i y_{k}\left(\sigma_{1}, \ldots \sigma_{n}\right)
$$

le variabili reali $\sigma_{\mu}$ con valori vicini complessi e coi loro conjugati

I punti

$$
\bar{t}_{\mu}=\sigma_{\mu}-i \tau_{\mu}, \quad t_{\mu}=\sigma_{\mu}+i \tau_{\mu} .
$$

$$
z^{\prime}=x(\bar{t})+i y(\bar{t}), \quad z^{\prime \prime}=x(t)+i y(t)
$$

cosi trovati riempiono nello $S_{2 n}$ due regioni (identiche) a $2 n$ dimensioni, includenti il $C_{n}$, e si corrispondono nella riflessione cercata.

$\mathrm{E}$ chiaro che già nel gruppo sinettico tutte queste riflessioni equivalgono al conjugio

$$
X_{k}=x_{k}, \quad Y_{k}=-y_{k},
$$

che è la riflessione determinata dallo spazio ordinario

$$
y_{\mathrm{x}}=0, \ldots \quad y_{n}=0 .
$$

$\mathrm{Ma}$ i teoremi precedenti ci conducono ad un punto di vista più elevato: 
XVII. Una qualunque v'arietà $V_{n}$ REALE ED ORDINARIA determina univocamente una trasforma sformazione sono le immagini dei punti complessi della $V_{n}$. Se la $V_{n}$ ba tratti reali $S_{n}$, $S_{n}^{\prime}, \ldots$, contenenti $i$ campi elementari $C_{n}^{\mathrm{I}}, C_{n}^{2}, \ldots$, tutte le riflessioni determinate da questi sono contenute nell'unica trasformazione simmetrica individuata dalla $V_{n}$.

La riflessione determinata da un qualunque campo elementare dello spazio $S_{n}$ potrd̀ proseguirsi analiticamente, pel tramite di coppie di punti reali. Questa continuazione analitica reale comprende non solo tutte le riflessioni, individuate (nei loro intorni) dagli altri campi elementari dello stesso $S_{n}$, ma anche quelle che vengano determinate dai campi elementari d'un qualsivoglia altro spazio $S_{n}^{\prime}$, non ostante il fatto che questi spazi sono connessi colla $S_{n}$ solamente pel tramite di punti immaginari.

Tutte queste proprietà sono invariabili rispetto al gruppo sinettico ampliato. Quindi esse devono ritrovarsi nella teoria delle $V_{2 \rho}$ generiche. Arriviamo cosi, per esempio, ad una generalizzazione del Teorema XV:

XVIII. Per stabilire una qualsiasi trasformazione puntuale sinettica o antisinettica fra due $S_{2 \rho}, S_{2 \rho}^{\prime}$ sinettici, basta ricavarne due regioni regolari di campi $C_{\rho}, C_{\rho}^{\prime}$ ORDINARI, $e$ farle corrispondere analiticamente.

Segue il corollario che tutti i $C_{\rho}$ ordinari equivalgono al campo

$$
y_{1}=0, \ldots y_{n}=0, \quad x_{\rho+1}=0, \ldots x_{n}=0 .
$$

Ma questo campo o spazio contiene solamente dei campi e spazì ordinari. Dunque:

Tutti gli spazi portanti un campo elementare ordinario, sono ordinari.

Nello stesso modo potremmo generalizzare i Teoremi XVI, XVII, ed anche pervenire ad ulteriori generalizzazioni. Applichiamo invece il nostro metodo a provare il teorema di Levi-Civita.

Designiamo con $\xi$ e $\psi$ due funzioni analitiche reali dei parametri $\sigma_{\mathrm{r}}, \ldots \sigma_{n}$ aventi un campo comune di esistenza $\{\sigma\}$. Continuiamo queste funzioni nel vicino campo complesso $\{t\}$, e designiamo con $u$ e $v$ le funzioni complesse risultanti. Se $z=x(\sigma)+i y(\sigma)$ è una regione ordinaria riferita allo stesso campo $\{\sigma\}$, o ad una parte $\{\{\sigma\}\}$ di esso, $z=x(t)+i y(t)$ sard lo $S_{2 n}$, o una regione di esso. La funzione complessa

$$
w(t)=u(t)+i v(t)
$$

si ridurrà per i punti della regione data [corrispondenti al campo $\{\{\sigma\}\}]$ ai valori

$$
w(\sigma)=\varphi(\sigma)+i \psi(\sigma) \text {. }
$$

Dunque:

XIX. Per determinare completamente una qualsivoglia funzione $w=u+i v$ delle variabili complesse $z_{\mathrm{I}}, \ldots z_{n}$, basta conoscere $i$ valori della funzione nei punti di una qualunque regione ORDINARIA $a$ n dimensioni, purchè questa entri nel campo di esistenza della funzione $w$.

$I$ valori $\phi$ e $\psi$, a cui si riducono le funzioni reali $u$ e $v$ nell' intorno d'un punto 
della regione data, possono essere prefissati come fun ioni analitiche ARBITRARIE dei parametri rappresentativi della regione.

Ne viene il corollario che $w=0$, se $\varphi=0, \dot{\psi}=0$.

Arriviamo più brevemente alla stessa conclusione partendo dal Teorema II, sostituendovi $n$ e $p$ con $n+\mathrm{I}, r$ con $n$, e $z_{n+1}{ }^{\cdot}$ con $w$.

Naturalmente, l'arbitrarietà nella scelta delle funzioni $\phi$ e $\psi$ deve prendersi colle debite precauzioni. Esse devono avere un campo comune di esistenza nella regione considerata. Se $i$ valori della funzione $u+i v$ sono conosciuti in una regione regolare generica (a $r$ dimensioni), la funzione rimane determinata completamente o in parte, cioè per una regione d'un $S_{2 \rho}$ sinettico ben determinato, ma non fuori di questo spazio. Se la regione data non è ordinaria, cioè, se la sua dimensione $r$ supera il numero $p, \mathrm{i}$ valori ridotti $\varphi$ e $\psi$ non possono scegliersi in modo arbitrario.

Ecco infine un complemento:

$\mathrm{XX}$. Se $i$ valori ridotti $p+i \psi$ della funzione $w$ (di cui parla il teorema precedente) sono reali $(\psi=0)$, la funzione deve assumere valori conjugati $u-i v$ e $u+i v$ nelle coppie di punti $z^{\prime}, z^{\prime \prime}$, che sono determinate dalla regione assunta.

(Veggansi i Teoremi XVI e XVII).

Ciò vale, naturalmente, in quanto coincidono i campi di esistenza della funzione e delle dette coppie, le quali devono proseguirsi insieme (pel tramite degli stessi punti) partendo dai punti della regione data e dai valori assegnati $₹$ e $\psi(=0)$.

Tali sono i teoremi generali che volevo sviluppare. In una Nota complementare in parte storica - mi propongo di trattare di casi speciali e di applicazioni geometriche.

Firenze, 23 marzo 1906.

E. STUDY. 